\title{
Téoros
}

Revue de recherche en tourisme

\section{Le Casino de Charlevoix}

Un équipement touristique?

\section{Claude Gingras et Lise Lapointe}

Volume 17, numéro 1, printemps 1998

Le tourisme dans un Pays à part : Charlevoix

URI : https://id.erudit.org/iderudit/1072374ar

DOI : https://doi.org/10.7202/1072374ar

Aller au sommaire du numéro

Éditeur(s)

Université du Québec à Montréal

ISSN

0712-8657 (imprimé)

1923-2705 (numérique)

Découvrir la revue

\section{Citer cet article}

Gingras, C. \& Lapointe, L. (1998). Le Casino de Charlevoix : un équipement touristique ? Téoros, 17(1), 33-39. https://doi.org/10.7202/1072374ar d'utilisation que vous pouvez consulter en ligne.

https://apropos.erudit.org/fr/usagers/politique-dutilisation/ 


\section{tan. \\ Le Casino de Charlevoix \\ UN ÉQUIPEMENT TOURISTIQUE?}

\author{
Claude Gingras, président, et Lise Lapointe, directrice générale \\ Exploracom inc., Cap-à-l'Aigle
}

En juin 1994, soit moins d'un an après l'ouverture du Casino de Montréal (octobre 1993). la Société des casinos du Quebec inaugurait, dans un bätiment (1929) situé sur les terrains du Manoir Richelieu, sa deuxième maison de jeu, le Casino de Charlevoix.

Le ministre des Finances de l'epoque, M. André Bourbeau, concluait la cérémonie d'ouverture par ces termes: a... le casino insuffle un dynamisme nouveau. Il diversifie un secteur touristique déja fort atrayant.. C'est par la conjugaison de la splendeur des décors de Charlevoix, de la chaleur de ses résidents de même que de la diversite de ses structures d'accueil et de divertissement, cest la conjugaison de tout cela, disje, qui bâtira une industrie capable de soutenir le développement économique durable. Le gouvernement du Québec est heureux de s'associer à ce développement. Longue vie au Casino de Charlevoix et prospérité à son industrie touristique. "s'

Par ces propos, le ministre soulignait les acquis de Charlevoix: ses paysages physiques et humanises. la tradition d'accuell de sa population, son infrastructure d'atractions et de soutien, érigée au fil des ans, et leur résultante, la cohésion entre le pays habité et le pays visité. Il décrivait aussi l'arrivée du casino comme un élement complementaire à l'offre touristique charlevoisienne, de nature à contribuer au développement touristique et économique de la région.

Dans le rapport Bertrand, un examen des possibilités d'exploitation de casinos au Québee datant de décembre 1979, on souligne que la légalisation des jeux de casino s'appuie historiquement sur une conjugaison de motifs, que voici: aaugmenter les revenus de l'État, stimuler l'industrie touristique, générer des impacts économiques, lutter contre les activités illégales et enfin, stimuler l'animation du milieu ${ }^{2}$. Plus loin, on ajoute: «En consequence [de l'analyse de ces motifs pour le Québec], on peut pratiquement considérer que le seul motif invocable pour justifier la législation des jeux de casino au Québec est l"impact touristiques. .

Aujourd'hui, quatre ans après l' inauguration, nous pouvons et nous devons poser un regard critique sur cet equipement et examiner ici sa véritable contribution à l'industrie touristique de Charlevoix et son influence sur l'orientation globale du développement touristique de la région. Et nos observations (voir l'encadré méthodologique) nous amènent à formuler l'hypothèse que le Casino de Charlevoix est à l'origine d'un clivage de clientè les qui compromet la viabilité des entreprises touristiques traditionnelles et imprime au développement touristique une direction qui s'ecarte résolument du chemin trace en plus de cent ans. A l'heure où chacun cherche à conquérir les marchés internationaux, Charlevoix, région du Québec au potentiel certain pour ces marchés, est-il en train de renoncer à ses atouts?
Nous nous pencherons d"abord sur des concepts quí conditionnent l'activité touristique, sur les notions de touriste, de milieu visitế, de relation d'échange entre visiteurs et visités, sur le respect des valeurs intrinseques du milieu dans le développement touristique et sur l'univers des perceptions d'un lieu à visiter afin de voir comment s'intègre le casino à la trame spatiale de Charlevoix et quelle est son influence sur l'industrie dans une vision prospective de développement. ${ }^{4}$

\section{LA CLIENTĖLE TOURISTIQUE TRADITIONNELLE}

Avant même de parler d'industrie touristique, de marketing, de marchés à conquérir et de rentabilité, il y a lieu d'examiner le noyau autour duquel s'organise cette industrie, c'est-ă-dire l'individu, celui qui part, qui rompt avec la vie wordinaires, pour deux jours, une semaine, un mois. Qu'est-ce qui le motive, l'incite à se déplacer, qu'est-ce qu'il recherche dans I'experience touristique? Il y a lieu aussi de tenir compte de la perspective de celui qui le reçoit.

Charlevoix est connu depuis longtemps comme région touristique. Déjà au milieu du $\mathrm{XIX}^{c}$ siècle ${ }^{5}$, à bord de magnifiques abateaux blancss, les estivants arrivent en masse à Murray Bay ${ }^{6}$ pour donner naissance à une villégiature cossue dans. la région. Le tourisme ici, prenant appuí sur ce passé de villég giature, s'est imposé de lui-mème, contrairement au développement délibéré de ce secteur d'activité que d'autres régions du Québec ont pu embrasser plus récemment. Les gens de ce pays ont grandi dans l'acceptation du 
passage de l'autre, le temps qu'il vienne chercher ce que la région a de micux à lui offrir, cette splendeur du paysage, cette réalité géographique qui loge au coeur même de l'identité de l'occupant.

Le Charlevoisien se retrouve aussi dans le regard de l'autre, de ce touriste qui observe, qui découvre, il y perçoit des bribes de sa propre réalité. Le lien s'établit, l'accueil est sincère. Le paysage prend une dimension culturelle dont l'expression se révèle dans l'art traditionnel et contemporain. Dans l'échange et la communication chacun trouve son compte. Le Charlevoisien parle et exprime son sgenre de view et apprend aussi de l'autre. Le touriste pour sa part découvre les différences, les particularités culturelles et en arrive à mieux saisir ce qui le distingue. à mieux se définir.

Certes, tous les touristes ne voyagent pas de cette façon. Tous ne possedent pas les mêmes valeurs. Selon leur personnalité, leur culture, ils ont des besoins différents. Ils recherchent des expériences touristiques qui correspondent à leurs valeurs et ils choisiront en conséquence leur destination de voyage. $D^{\prime}$ ailleurs, du moins jusqu'à récemment, la région touristique de Charlevoix a toujours evoqué le paysage? , jusque dans l'organisation même des attraits et des éléments de soutien, qui permettent au visiteur d'explorer cet univers en fonction du degré recherché de dépaysement. Celui-ci pourra l'aborder comme simple observateur ou pousser plus loin la découverte et établir un contact lui permettant de mieux comprendre le milieu ou encore approfondir l'echange et vivre une expérience touristique de nature à le combler sur le plan personnel. Mais la notion de paysage physique et humanisé, la quête de sensations et d'émerveillement demeurent le fondement de l'expérience touristique charlevoisienne.

Par conséquent, il y a concordance entre la clientêle touristique de la région et l'élément attractif que représente le paysage. En 1986, le tourisme d'agrément de sćjour et de circuit constitue à lui seul près de $85 \%$ du marché de Charlevoix ${ }^{3}$. Le Plan de développement touristique de Charlevoix définit le tourisme d'agrément comme cun voyage où la découverte de la région, le repos, la relaxation et le dépaysement sont les motifs essentiels... Il s'agit en fait du tourisme 'classique', sans but spécifique. $x^{\text {"E }}$ En outre, dans ce document, on remarque la relation qui s'établit entre la perception du lieu et sa consommation. ${ }^{11}$

\section{L'ARRIVÉE DU CASINO ET D'UNE NOUVELLE CLIENTÈLE}

Le message véhiculé au lancement du Casino de Charlevoix faisait de ce demier un important instrument de développement, qui diversificra l'offre touristique et entraînera une augmentation du nombre de visiteurs dans la région. Retenons de cet énoncé deux éléments qui méritent une attention particulière: le terme «visiteurs, d'une part, et la présomption de compatibilité des clientêles, de l'autre.

Il est difficile d'avancer avec certitude qu'on ne peut comptabiliser dans la clientèle touristique les visiteurs attirés par le casino. Cependant, dans la mesure où, comme le souligne le sociologue Marc Laplante,

"pour degager la signification première du voyage d'agrément, il faut latter contre les vues dominatrices d'une industrie qui, pour se faire grosse et puissante, appelle touristiques toutes sortes de deplacements (tourisme d'affaires, de congrès, de sante, religieux, etc.) et contre celles des administrations publiques qui, sur ce sujet particulièrement, ne font qu'enteriner les vues de l'industrie puisqu'elles ne considerent que les retombées économiques du fourisme dans leurs coffres "n,

on peut considérer que le terme «visiteur» tient lieu ici de fourre-tout regroupant

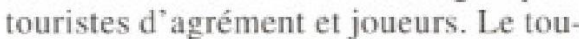
riste d'agrément a ceci de particulier qu"il se deplace pour le plaisir, pour l"agrément du voyage, alors que le joueur se déplace essentiellement pour se rendre au casino.

A l'occasion d'une conférence de presse en 1979 sur l'exploitation des casinos au Québec, le ministre des Finances d'alors, M. Jacques Parizeau, mentionnait que «le casino n'est pas implanté parce que c"est une machine à faire de l'argent mais il est implanté dans une région touristique parce que cela attire des joueurs pour les transformer en touristes, donc cela augmente le potentiel touristique d'une région $*^{12}$. Objectif tout à fait louable sauf que l'ob- servation nous invite â conclure â l'inverse. ${ }^{13}$ Le casino ne transforme pas de joueurs en touristes mais il profitera i l'occasion du passage du touriste d'agrément: un petit $24,00 \$$ en 1994 , un petit $30,35 \$$ en 1997 , un petit $34,00 \$$ en $1998^{14}$, des petits qui ne font plus de petits dans la région.

Toutefois, par souci de rentabilité, le casino ciblera le client susceptible de passer plus de temps dans l'établissement. done moins de temps dans la région, celui qui se déplace dans le but premier de jouer. Dans un mémoire, I'ancien Office de planification et de développement du Québec (OPDQ) mentionne que «si l'on considère le casino comme un instrument de développement touristique, il ne faut pas oublier que ce ne sont pas tous les touristes qui sont intéressés par le jeu ou ont les moyens d'y participer ${ }^{15}$.

La clientèle traditionnelle de Charlevoix a-t-elle le profil d'une clientèle intéressée par ce type de produit? L'OPDQ indique à ce propos dans son mémoire:

* Si l'on chenche à préciser parmi ces categories lamateurs de plein air, hedonistes, adeptes du soleil, amateurs de choses culturelles / les clients les plus susceptibles d'être artires par les casinos el d"avoir les moyens d'y jouer, il nous semble devoir exclare à prime abord les amateurs de plein air qui en moyenne sont moins fortunés et surtout exencent leurs activités récréatives dans la grande nature, loin des centres à la mode el très achalandés. À quelques exceptions près, il ne semble pas que les amateurs de choses culturelles solent non plus des clients importants pour les casinos so

A l'inverse, selon nos observations et les propos recueillis pendant notre enquête, la clientèle du casino ne semble pas intếressée par le produit touristique traditionnel de Charlevoix. Elle n achète pas dans les galeries d'art ni dans les boutiques d'artisanat et de souvenirs, ne se livre pas à des activités comme les croisières aux baleines, ne fait pas de ski, ne va pas au concert et, somme toute, participe tres peu à l'économie touristique de Charlevoix. 
Le directeur général du casino, M. André Loubier, souligne, en s'appuyant sur des ćtudes internes, que la marche représente la principale activité, autre que le jeu, des clients venus expressément pour le casino. Et il reconnait que le casino a rapidement abandonné une promotion visant a l'associer aux stations de ski de Charlevoix, la direction ayant constaté que le skieur passe la journée à la station, se couche tôt et n'a guère le temps de jouer. sTout le monde fait des erreurs dans sa vie., ${ }^{17}$

La nature et la culture constituent pourtant le fondement de l'industrie touristique de la région. La particularité du produit touristique charlevoisien, sa spécificité, c'est le pittoresque, l'aventure, le dépaysement, qui s'expriment dans les divers attraits et éléments de soutien développés au fil des ans et que le touriste avait l' habitude de découvrir avec fascination.

\section{LA CLIENTĖLE DU CASINO}

Ces propos nous amènent à décrire brièvement certains éléments du profil de la clientềle du Casino de Charlevoix. En ce qui concerne la provenance de cette clientêle. il est bon de rappeler qu"en mars 1982, M. Michel Hupé, auteur d'une analyse comparative de deux rapports sou= mis par la Société des loteries et courses du Québec (SLCQ) au ministre des Finances (mars 1981) et par le ministre du Revenu au Comité ministériel permanent du développement économique (février 1982), souligne que sles deux rapports s'accordent sur le fait que les jeux de casino contribueraient à augmenter le nombre de visiteurs au Québecs ${ }^{18}$. Or si l'on examine les rapports annuels présentés par Loto-Québec, l'on constate que la clientèle hors Québec du Casino de Charlevoix, situé en pleine région touristique, représente respectivement $3,38 \%, 2,31 \%$ et $2,60 \%$ du nombre total de visites pour les années 1994-1995, 1995-1996 et 1996-1997. ${ }^{19}$

L'auteur de l'analyse croit bon lui aussi de cortiger les données des deux rapports relatives à la contribution des non-résidents, qu'il juge trop optimistes.

* La grande majorité des experts europeens rencontrés lors de visites sur le terrain affirment en effet que leurs entreprises ne seraient pas viables financienemen sans la clien- tele réguliere qui provient des bassins de population des environs. c'est-à-dire à moins de une à deux heures de voinure du casino..$^{20}$

Dans Charlevoix, les données sur la provenance de la clientèle tendent à appuyer les remarques de M. Hupé. La clientèle du casino provient à $50 \%$ de la région de Québec métropolitain et Beauce-Appalaches, à 7-10\% de la région immédiate, a $12 \%$ du Saguenay-Lac-Saint-Jean et a 20 $25 \%$ du reste du Québec et principalement de la région de Montréal. C'est done dire que $72 \%$ de la clientèle du casino réside à moins de deux heures de route de l'établissement. ${ }^{21}$

La proximité de cette clientèle favorise l'aller au casino mais également le retour à la région de provenance, ce qui limite la durée du séjour ét la consommation des divers attraits de Charlevoix. Selon le directeur général du Casino de Charlevoix, on peut qualifier une très grosse partie de la clientèle de clientele d'autocar, en grande partie répétitive. ${ }^{22}$ Une clientèle qui, selon le forfait, a le transport payé par le casino à la condition $\mathrm{d}^{+} \mathrm{y}$ passer un nombre minimal d'heures. L'autocar arrive tôt le matin aux portes de l'établissement et repart en fin d'après-midi. Des autocars qui reviennent, certes, mais toujours pour le casino. Des entrées comptabilisées dans les statistiques comme des visiteurs... comme des touristes.

Selon le discours officiel, l'arrivée du casino dans Charlevoix devait avoir comme

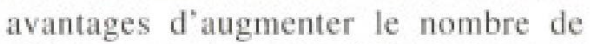
visiteurs, car on y accueillerait des gens qui, de toute façon, ne venaient pas ou ne viendraient pas dans la région, et de contribuer ainsi à l'essor de l'industrie touristique. Mais si les chiffres s'additionnent, il y a lieu d'examiner si les individus en font autant. Voici qu'interviennent les notions d'incompatibilité et de clivage de clientèles.

\section{INCOMPATIBILITÉ ET CLIVAGE DE CLIENTĚLES}

Le clivage de la clientèle résulte de l'incompatibilité de comportement des individus qui la composent, d'une part, et de l'orientation divergente que prend le développement touristique depuis l'arrivée du casino dans la région, d autre part. Nos observations nous permettent d'avan- cer que la nature du comportement de la nouvelle clientèle intervient dans lá qualité de séjour recherchée par la clientèle traditionnelle, ${ }^{23}$ Insatisfaite, celle-ci nous laisse savoir, ả répétition, qu'elle délaissera Charlevoix comme destination de voyage.

Les activités du casino n"entraînent pas d'augmentation de la clientèle touristique, pour deux raisons. D'abord, le comportement d'achat du joueur differe totalement de celui du touriste traditionnel et on ne peut de ce fait catégoriser le joueur dans la clientèle touristique. Il faudrait créer une catégorie distincte, en prenant appui sur une étude approfondie de ce comportement d'achat. Ensuite, le joueur a tendance à faire fuir le touriste traditionnel. Nous en avons des témoignages fréquents et nous assistons non pas à une augmentation mais à une substitution de clientêle. Le processus est entamé et, compte tenu de la domination qu" assure au Casino de Charlevoix son énorme pouvoir de promotion, il devrait se poursuivre inexorablement. ${ }^{24}$

Par surcroit, la concurrence entre les deux clientèles, suscitée par le casino, influe sur l'orientation du développement touristique. La nouvelle clientele imposera des produits, des equipements, des éléments de soutien d"une autre nature, incompatibles avec ceux que recherche la clientèle traditionnelle. Il en découlera une modification du paysage, de la marque de Charlevoix, qui ne fera qu'exacerber l'insatisfaction et, par conséquent, la fuite de la clientèle traditionnelle.

Examinons maintenant, dans la seconde partie de cette analyse, les incidences du casino sur l'organisation du territoire et la nature du développement touristique de Charlevoix.

\section{LES TERRITOIRES RÉEL, IMAGINAIRE ET À DÉCOUVRIR}

Selon nous, un développement touristique exemplaire devrait chercher à résister à la tendance à l'uniformisation des produits et à mettre en valeur et à protéger au contraire les particularités physiques et humaines de la région. Ce développement pourrait ainsi s'appuyer sur un modèle qui mettrait en relation trois représentations différentes d'un mème espace territorial. 
Tout d'abord le territoire réel, celui qui. compte tenu de son potentiel et de ses caractéristiques, a forgé les traits culturels de l'occupant. Dans Charlevoix, les éléments physiques de la mer, de la terre et de la forêt se sont traduits par une trilogie de paysages humanisés: des villages maritimes, des villages agtaires, des villages forestiers où se sont enracinés le geste, la coutume et la vie en un riche héritage, en une mémoire collective que le touriste vient goutter, des couleur's de laquelle il vient s' imprégner, le temps d'un séjour. A travers sa propre culture, il posera un regard sur le territoire à découvrir.

Ce deuxième territoire correspond au territoire aménagé, celui qui propose au touriste les outils nécessaires à la découverte. Lauberge traditionnelle, où vivre une nouvelle expérience culinaire, les économusées, les centres d'interprétation, les galeries d'art feront partie de ces outils. Le touriste traditionnel, en bon explotateur, choisira les routes pour ce qu'elles ont à raconter: une vue saisissante de la mer, un village fleuri au printemps, la lumière de la vallée. Le temps prendra ici le rythme qu'il voudra lui donner, au gré des paysages. L'aménagement touristique du territoire à découvrir, expression pour le visiteur du territoire réel, tracera les prémisses d'une bonne communication entre les deux groupes, touristes et oceupants. Le territoire à decouvrit possède des liens étroits aussi avec un troisieme territoire, le territoire imaginaire.

Dans le territoire imaginaire naissent le goût du voyage, le rêve du voyageur. Ce dernier y esquissera à loisir, en fonction de ce qui le touche, les formes, les rapports souhaités. La lecture d'un beau livre, le témoignage d'un ami, un récit ancien seront de ces éléments qui nourrissent l'univers des perceptions, cet univers auquel il aura donné vie à sa façon avant le véritable départ. Et cette évocation de Charlevoix, de ses paysages, de sa tranquillité, doit trouver sa consécration dans l'aventure et le voyage. Si le touriste rêve de tranquillité, l'occupant, en bon hôte, doit pouvoir le satisfaire.

\section{LE CASINO ET LA PROBLÉMATIQUE D'INTÉGRATION}

L'arrivée du casino comme nouvel équipement, comme nouvel et puissant acteur

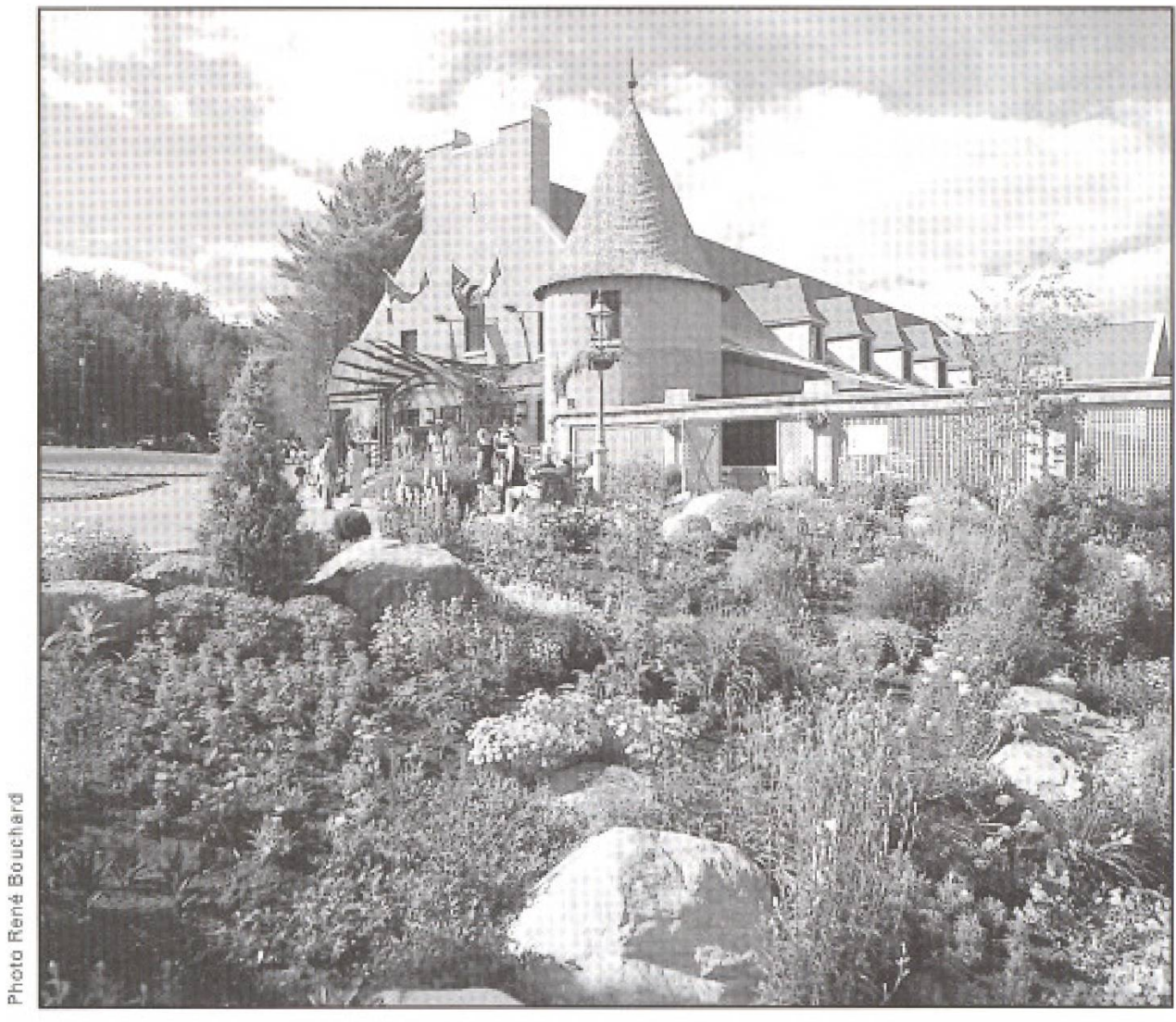

de l'industrie stouristique* de Charlevoix, a des conséquences sur le territoire imaginaire, compte tenu de son influence sur la perception de la tégion, ainsi que sur le territoire à découvrir, compte tenu cette fois de son intégration à la trame spatiale de Charlevoix. Ajoutons que les activités du Casino de Charlevoix se répercutent nécessairement aussi sur le territoire réel, en particulier sur le tissu social et sur l'économie de la région dans son ensemble. Ces aspects, bien qu"importants. débordent toutefois le cadre de la présente analyse. ${ }^{26}$

\section{LE CASINO ET L'IMAGE DE CHARLEVOIX}

Jusqu'a récemment, le paysage, dans son sens large, représentait l'unique évocation de Charlevoix comme destination touristique. Depuis 1994, la région se transforme en destination de jeu et elle lance désormais un message qui s'adresse à un tout nouvel imaginaire, un message dominant car le budget de promotion d'une société d'Etat est sans commune mesure avec celui de n'importe quel autre intervenant touristique de Charlevoix. Dans son anaIyse comparative, Michel Hupé tire d'études de marché et de ses propres observa- tions la conclusion que al'on ne pourrait attirer le touriste principalement non résident dans des centres de villégiature éloignés des grands póles touristiques de Montréal et de Québec qu'en imprimant une très forte intensité à l'exploitation du jen. $x^{26}$

Bien sûr, le casino se targue de toujours mettre en valeur la beauté de la région dans sa publicité mais ce message, semblable du reste à celui de l'Association touristique régionale (ATR $)^{27}$, qui ne manque jamais de mettre en valeur le casino, pèche par incohérence puisqu 'il s"adresse à deux clientèles à l'impossible cohabitation. Et ce message incohérent fait aujourd'hui l'objet d'une diffusion d'une ampleur encore jamais vue, visant en particulier les marchés internationaux.

\section{L'INFLUENCE DU CASINO SUR L'ORGANISATION SPATIALE DU TERRITOIRE}

Le casino attire, observons-nous inévitablement, une clientèle peu portée vers des activités comme les visites de musée, de galeries d"art, les croisières en bateau. En contrepartie, cette clientèle demande des équipements qui répondent à ses besoins 
propres. L'organisation de la destination de jeu devra leur permettre de circuler en vitesse sur la route, de manger en vitesse dans des restaurants uniformisés et sans cachet. En bons hôtes, nous devons aussi satisfaire ces besoins.

Depuis quatre ans, on met ainsi l'accent sur le réseau routier: des routes de plus en plus larges, de plus en plus planes pour Charlevoix, pays de mer et de montagnes. Aux abords de ce réseau apparât une restauration nouveau genre, dont l'affichage, par manque évident de planification et de sensibilisation, n"a pas fait l'objet de toute l'attention qu'il exige.

Le casino amène aussi des problématiques architecturales. Au coeur de quartiers résidentiels, on assiste à l'apparition de nouveaux commerces, au style acondow dont on questionne encore le sens en région rurale. On assiste aussi à une prolifération des mises en vente de résidences sur le boulevard des Falaises, témoignage de la villégiature charlevoisienne, où l'on venait chercher calme et tranquillite, aujourd'hui chemin d'accès, à sens unique, au casino.

Cette modification du paysage ne peut que $\mathrm{s}^{\dagger}$ accentuer car les entreprises touristiques traditionnelles, délaissées par la clientèle traditionnelle et négligées par lá clientèle joueuse, auront de la difficulté à survivre. Bon nombre des entreprises touristiques avec lesquelles nous avons eu des entretiens libres (voir l'encadré méthodologique) font état, déjă, d "une diminution de revenus de l'ordre de $30 \%$. Ces entreprises, si elles ne peuvent plus assurer leur rentabilité, devront cesser leurs activités ou adapter leurs produits à la nouvelle demande. Comme le déclarait à l'occasion d'une tribune téléphonique télévisée l'ancien préfet de la Municipalité régionale de comté (MRC) de Charlevoix-Est, aujourd'hui président du Comité de vigilance du Casino de Charlevoix, M. Jean Lajoie, ales galeries d'art, c'est tout simplement une adaptation, les gens vont se réadapter... Je suis convaincu que les personnes qui wont au casino ne sont pas nécessairement des gens qui vont acheter de l'art $x^{23}$. M. Lajoie venait confirmer par ce propos notre point de vue: nous assistons non pas à une intégration du casino à Charlevoix, en tant que produit touristique complémentaire, mais bien à une intégration de Charlevoix au casino, avec toutes les conséquences que cela suppose.

\section{LE DÉVELOPPEMENT TOURISTIQUE}

En novembre 1987, 1'Association touristique régionale présentait la version dé finitive du Plan de développement touristique de Charlevoix. L'ATR chapeautait l'équipe de travail mise sur pied pour l'élaboration de ce plan. L'équipe réunissait des représentants de plusieurs ministères et organismes dont les décisions influent sur le tourisme, des représentants des MRC de Charlevoix et de CharlevoixEst et des intervenants socio-écononiques de la région.

Le plan dégageait les principaux axes de développement de Charlevoix et proposait neuf grands projets lies au potentiel physique et humain du territoire. Au nombre de ceux-ci, mentionnons l'aménagement d'un parc marin national à l'embouchure du Saguenay, le développement du Massif de Petite-Rivière-Saint-Francois pour le ski, la création d'une maison de la culture à Saint-Irénée, la réalisation d'un circuit patrimonial intégré et l'amé= nagement d"une piste cyclable reliant Baie-Saint-Paul et La Malbaie. La plan mettait nettement l'accent sur wl'image la plus forte du produit touristique de Charlevoix, en I'occurence la beauté de ses paysages $\ldots, z^{29}$

En 1998, le produit traditionnel de Charlevoix correspond à un marché en croissance à l'echelle internationale. Ainsi, selon la Commission canadienne du tourisme (CCT).

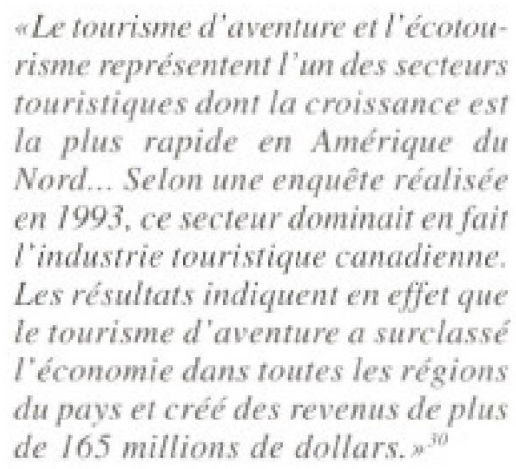

Dans une autre étude du tourisme d'aventure, publice par la CCT en 1995, on retient des points saillants que les gens de l'extérieur, presque tous des Américains et des Européens, représentent $44 \%$ des touristes d'aventure au Canada. Quant aux Canadiens, ils composent $56 \%$ de la clientèle ${ }^{31}$ Le pourcentage de $44 \%$ s'applique non pas à une clientêle hors Québec, comme les plus ou moins trois pour cent mentionnés plus haut pour la clientèle du casino, mais à une clientèle hors Canada. Voilà des données intéressantes pour qui convoite les marchés internationaux.

Autre point saillant de l'étude, la durée moyenne des forfaits de tourisme d"aventure était de 5,3 jours, et leur prix moyen de 143 dollars par jour. Plus de $75 \%$ des excursions d'aventure au Canada ont duré plus d'une journée., Voilà également des données intếressantes pour qui cherche à prolonger le sejour des visiteurs dans Charlevoix.

Enfin, dans un autre document de la CCT, on lit que le marché du tourisme culturel et patrimonial est en croissance lui aussi. a Sa croissance est évaluée à 15 p. 100 par année et le Canada doit être en mesure de répondre à la demande mondiale en matière de tourisme culturel pour demeurer concurrentiel sur la scêne touristique internationale. m $^{33}$

D'après nos démarches auprès de Tourisme Québec et de Loto-Québec, le gouvernement n'a réalisé aucune étude dẹ l'incidence des casinos sur l'industrie touristique après l'ouverture de ces derniers. Il n'y a eu que des études prévisionnelles, qui semblent avoir nettement su= restimé ou mal jaugé les retombées touristiques de l"exploitation d'un casino pour avoir présumé, dans le cas de Charlevoix, de lá compatibilité de la clientẻle traditionnelle de la région et d'une clientèle de joueurs ainsi que de la compatibilité de la vocation touristique de Charlevoix et de la vocation de son casino. Or la remarquable persistance de nos observations depuis près de trois ans rend aujourd'hui de telles études impérieuses et urgentes.

Si ces recherches, menées de façon independante, devaient confirmer notre hypothese, elles nous imposeraient un choix entre les avenues de développement que propose le casino et celles qu'offrent les marchés du tourisme d'aventure et de l'écotourisme et du tourisme culturel et patrimonial et par la mểme occasion l'Eclaireraient.

Le débat qui s'engage doit s'étendre à l'ensemble des intéressés, y compris la population touchée par le développement de Charlevoix, quelle qu'en soit la nature. Ici le tourisme a eté essentiellement l'ex 
pression de notre mémoire collective. II nous a permis aussi d'acquérir un savoirfaire que nous devrions mettre à profit plutôt que de lui tourner le dos.

Charlevoix, c'est un pays en soi, une terre que le temps a façonnée, Iui consacrant sa différence. Nous devons nous demander aujourd'hui à quel pays nous sommes redevables et envers quel pays nous sommes responsables. Nous devons nous demander aussi quel pays nous voulons nous donner.

Un comité de lecture a lu et accepté ce texte

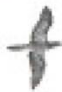

\section{NOTES}

1 Nones pour une allocution du ministre des Finances, Monsiew Andre Bourbau, à loccasion des céremonies d'ouverture du casino de Charlevoix, Bibliothèque de l'Assembléc nationale du Québec (1994), Québec, juin, (non publis), p. 3.

2 Société des loteries et courses du Québec (1979), Groupe d'analyse et de recherche. Rapport Bertrand: Le domaine des jeux de casino, préparé par Roger Bertrand, (non publié), p. 4.

3 bid., p. 22.

4 II convient de noter que nous n'abordons pas dans ce texte les motifs du choix de Charlevoix comme emplacement du casino par le gouvernement du Québec car cet aspect pourrait à lui seul faire l'objet de toute une analyse.

5 La seconde moitie du XIX" sieccle se caractérise par un accroissement des raisons pour voyager. in Laplante, Marc (1996), L'experience touristigue comlemporaine: Fondements sociaur et culturels, collection: * Tourisme* (Sainte-Foy: Presses de l'Université du Québecl. p. 28

6 Du nom du gouverneur James Murray, qui, après la conquêtte, divise l'ancienne seigneurie de La Malbaic en deux parties, qui porteront les noms de Mount Murray, à l'est de la rivière Malbaie, et de Murray's Bay, à l'ouest. Le toponyme de Murray Bay ne prendra jamais racine dans la population locale.

7 On note aünsi à la page 33 du Plan de dévetoppemen louristique de Charlevoix: Rapportfinal, Association touristique régionale de Charlevoix, Charlevoix, 1987, que la beauté des paysages vient au premier rang des aspects de la région particulièrement bien perçus par les visiteurs. Viennent ensuite I'hospitalité des gens et la beauté des villages.
8 Association touristique régionalle de Charlevoix (1987). Plan de developpement torristique régional: Rappon final, Charlevoix, p. 83.

9 bid, p. 82.

10 Wid, p. 32 et 33

11 Laplante, Marc (1996), Lexperience towristique contemporaine: Fondements sociaur et culturels, collection: *Tourismes (SainteFoy: Presses de l'Université du Québece), p. 41 .

12 Conference de presse de Monsicur Jacques Parizeau, Bibliotheque de l'Assemblée nationale du Québec, Québec, avril 1979 (non publiể), p. 1 .

13 Les cas pathétiques sont nombreux. Citons celui d' un couple avee un enfant, qué nous rapporte l'un des sujets interrogés, venu passer une semaine dans la région. Le premier jour, la femme et l'enfant vont faire de l'équitation, pendant que l'homme va jouer. A la fin de la joumée, le couple repart fauché.

14 Dépense moyenene par visite effectuée au Casino de Charlevoix, touristes d'agrément et amateurs de jeu confondus. Données provenant d'une entrevue accordée par le directeur général du Casino de Charlevoix, M. André Loubier, à la Têlévision communautaire Vents et Marécs, Cap-à-1'Aigle, et diffusée le 18 février 1998 .

15. Ofice de planification et de développement du Québec (1978), L'implantation de casinos et le développement reglonal: Memoire presente par l'OPDQ au Groupe interministeriel d'efude sur l'implantation de casinos. au Québec, Québec, août (non publié), p. 2.

16 hid., p. 5.

17 Propos de M. André Loubier recueillis à l'occasion de l'entrevue mentionnéc à la note 14.

18 Hupé, Michel (1982), Société des loteries et courses du Québec, Les jeux de casino an Québec: Une analyse comparative de contenu, Québec (nôn publié), p. 4.

19 Ces rapports annuels utilisent l'expression * nombre de visiteurss mais, après vérification auprès de M. Jean-Pierre Roy, porteparole de Loto-Québec, il s' agit du nombre de visites. La distinction est importante car un même visiteur peut fäire plusieurs visites.

20 Hupé. Michel, op. cil., p. 12.

21 Données recueillies à l'occasion de l'entrevue télévisêe mentionnée à la note 14

22 Propos de M. André Loubier recucillis a l 'occasion de l'entrevue mentionnée à la note 14.

23 Un de nos points de vente, une auberge, a institué un couvre-feu pour assurer la tran- quilité des lieux le soir et préserver sa clientèle traditionnelle. On y entre au plus tard à 23 h. Le casino ferme a 4 h. Nous avons recueilli de nombreuses autres observations en ce sens.

24 Dans un sondage présenté au Comité sectoriel touristique de Charlevoix (Zins Beauchesne et associés (1997), Etude des perceptions des clienteles à l'égard de la région touristique de Charlevoix: Rapport firal. Québec, décembre), on indique à la page 67 que, parmi les attraits, événements et spectacles les plus appréciés par les clien-

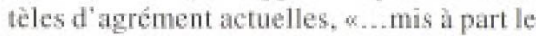
"Casino" qui est identifié par 16,5 o des répondants comme un attrait d'intérểt [sur 38 mentions], aucun autre cvenement n'est mentionné dans une proportion au-delà de 7 \%

25 Nous soulignons à l"intention des chercheurs et étudiants des deuxième et troisième cycles que les domaines et sujets de recherche liess au casino sont nombreux et promettcurs. Mentionnons, à titre d'exemples: la perte d"emplois causée par le jeu compulsif par rapport à la cráation d'emplois par le casino; l'incidence sur les retombêes économiques régionales de la déviation de la dépense touristique vers le casino en tenant compte de l'importante variable qu'est l'obligation pour le casino de verser, par l'entremise de Loto-Québec, un dividende au gouvernement.

26 Hupé. Michel, op. cis., p. 23.

27 Les issociations touristiques régionales (ATR) ont le mandat, entre autres, de réaliser la promotion touristique des diverses régions touristiques du Québec.

28 Propos de M. Jean Lajoie recucillis à l'émis= sion wQuébec en directs, diffusée par le Resenu de l'information (RDI) le 3 octohrc 1997.

29 Association touristique régionale de Charlevoix (1987). Plan de développement touristique régional : Rappont final, Charlevoix, p. 120.

30 Commission canadienne du tourisme (1997), Le tourisme d'aventure el lécolonrisme: Les enjeux, Ottawa, septembre (non publié), p. 3.

31 Commission canadienne du tourisme, Direction du Canada (1995), Le lourisme d'aventure au Canada: aperça du produit, du marche et du potentiel conmencial (Otawa: Commission canadienne du tourisme. juin), p. 47.

32 Ibid., p. 10

33 Commission canadienne du tourisme (1997), Réaliser le potentiel du tourisme culturel et patrimonial: Un résume des sir tables rondes régionales, Otawa, décembre (non publié). p. I. 


\section{BIBLIOGRAPHIE}

Remarque: pour éviter d'allourdir inutilement cette bibliographie, nous ne répéterons pas ici les sources documentaires citées dans les notes qui accompagnent le texte.

Assemblee nationale du Québec (1993). Procés verbaux a commission parlementaire des 3 et 4 juin 1993 sur l'érude du projer de Loi 84 / Loi sur la Régie des alcools, des courses et des jewx et modifant diverses disposition légiklatives], Position de la Süreté du Quebec, Québec, juin (non publié), $17 \mathrm{p}$.

Assemblée nationale du Québec (1993), Procèsverbaux: commission parlementaite des 3 el 4 juin 1993 sur l'étude du projel de Loi 84 I Loi sur la Régie des alcools, des courses el des jewx et modifiant diverses disposition legislatives/ Position da Service de police de la Communaule urbaine de Monträal, Québec, juin (non publié), $18 \mathrm{p}$

\section{MÉTHODES DE RECHERCHE}

Notre analyse s'appuie sur une recherche documentaire. sur des activides dobservation et sur deux séries d"entretiens.

\section{L'observation}

Notre société. Exploracom, offre les visites quidées du Manoir Richelieu. Devant notre poste, dans le grand hall face a la réceptian, passant taus les clients et visiteurs de Ithotel. Rappelans que le Manoir Richelieu est situd id deux pas du casino et, de ce fait, il constitue un lieu privilégié de rencontre des diverses clientẻles touristiques de Charlevoix:

Comme nous tenons des statistiques quotidiennes sur ces visites quidess, nous pouvons dire que nous avons assuré une présence persannelle sur les lieux / $c$ 'est l'un de nous qui átait alors sur place plutob́t qu'un de nos employés) de 2356 heures, réparties en 336 jours sur une période de deux ans ot huit mois (du 1* juillet 1995 au 28 février 1998)

De notre poste, pendant toutes ces heures, nous avons pu observer les comportements des visiteurs de même que I'evolution de ces comportements. Cette premièré étape a éveillé chez nous un interüt, qui nous a amenés, avec le temps, dे engager des discussians sur le produit tauristique de Charlevoix avec les gens qui dóambulaient lavec nas points de vente aussi; voir ci-dessous) et, de façon beaucoup plus assidue, avec les persannes qui cansommaient la visite guidée.

Ces observations des comportements des touristes avsient un caracture informel elles devaient appuyer nos décisions d'entreprise et nous ne pensions pas dै l'épeque qu'elles serviraient d'autres fins. Nous avons né anmoins réslisé une première synthảse des faits at gestes observés et des propos rec ueilis car nous tenians à approfandir l'investigation.

\section{L'entretien libre}

Notré sociêté a édité en décembre 1996 un ouvrage dont elle assure elle-même la distribution. Au courş de la saison estivale 1997, nous comptions, dans la région seulement, 41 points de vente, soit 19 dans l'ouest jusqu'd BaieSaint-Paul, in cluant I'lle-aux-Coudres, et 22 dans l'est jusqu' ì Tadoussag.
Comference de presse de Monsieur Genard D. Levesuae, Bibliotheque de l'Assemblé nationale du Québec, Quéhec, décembre 1992 (non publié). $31 \mathrm{p}$

Conférence de presse de Monsieur Paul Berthaume, Bibliothèque de l'Assemblée nationale du Québec, Québec, juillet 1976 (non publié). $36 \mathrm{p}$.

Horwath Consultant (1995), Etude d'impaet de l'implanation du casino de Hull, preparee pour la Ville de Hull, Quebee, mars/avril, $8 \mathrm{p}$.

Loto-Québec (1994-1997). Rapport annuel (Montréal: Loto-Québec).

Maciocia, Cosmo (1982). Deputê de Viger et porte-parole en matière de tourisme pour l' $\mathrm{Op}$ position liberale a l'Assemblée nationale, Les casinos an Quebec: Un dilemme socio-econowique, Montreal/Québec, mai (non public), 22 p.
Ministère du tourisme, de la Chasse et la peche. Direction générale du tourisme, Direction du développement touristique, Service de l'aide à l'industrie (1978). Proposinions sur I'mplamiation de casinos an Québec, Québec, aoùt (non publié). $197 \mathrm{p}$.

Sociélé des loteries et courses du Quebec (1982). Les jeur de casino au Quebec: L'exploiration des jêr de casino comme outil de developpemem rourishoue : Lear porice. leur linite. Quebec, juin (non public), $15 \mathrm{p}$.

Tourisme Québec. Direction de la recherehe et du développement (1997), Le towrisme au Oubber en 1995: Une realité economigae importanite (Québee: Tourisme Québec, mars), $102 \mathrm{p}$.
Sur ces 41 points de vente, 28 sont des entreprises qui vivent essentiellement du tourisme, les 13 autres vivant d'une clientêle mixte (locale et touristique). Nous avons visité ces points de vente un minimum de trois lois pen= dant la saison et avons eu des entretiens à au moins une accasion, et le plus souvent ả deux occasions, avec cha" cune des entreprises essentiellement touristiques. Nous awons eu quelques entretiens aussi avec les entreprises àclientèle mixte.

Nous qualifions ces entretiens de libres parce qu'ils ne s'inserivaient toujours pas dans une recherche afficielle et n'étaient précédès d'aucune demande đ'entrevue. Mais ces entretiens allaient plus loin que nos premiers travaus d'observation et ils se distinguaiant de ces derniers de deux façans. D'abard, un certain nambre d'entre eux ont eu lieu après que nous ayons été invités, fin aoūt 1997, à participer au prasent numero de Téoros. Ensuite, nous avions cette fais un abjectif plus précis, en l'ac currence verifier la validite des observations de la premiere étape et nous informer du rendement des entreprises depuis. l'auverture du casino. Nous cherchions en somme à acquérir l'assurance nocessaire a la formulation d hypotheses.

\section{L'entretien seat-directif}

Compte tenu des moyens très madestes dont nous disposions, nous ne pouvions envisager it cette etape une ennuhte quantitative aupres des exploitants touristiques de Charlevoix. Comme notre abjectif ici était d'accroïtre la glausibilite de notre hyoothese principale, nous avons élu de réaliser cinq entrevues de 90 minutes aver des dirigeants d'entreprises touristiques. Nous avons conçu un plan d'entrevue qui abordait les sept thòmes suivants a) le profil de l'entraprise; b) l'évolution de l'entreprise; c) la perception de la clientèlec d) la promotion touristique: e) la concurrence; fl l'évolution des revenus de l'entreprise: g) le casino et le développement touristique.

Nous avons ensuite dófini les critères de sablection nue vaici : les entreprises à interroger devaient aeuvrer essentiellament dans le secteur touristique, avoir débuté leurs activités avant rouverture du casino et représenter des produits differents des deux sous-groupes que sont les éléments de soutien (hábergement, restauration, otc.) el les élements d'attrait / boutiques de sauvenirs, econamusées, etc_l. Les produits représantás t́taient les suivants : I'auberge traditionnelle, le gilte touristique, l'attrait naturel, l'attrait cultural, la boutique d'artisanat.
Nous avons dũ communiquer avec sept entreprises car deux entreprises ont refusi de nous accorder une entrevue. malgré notre engagement à ne divulguer leur identité à quiconque. Dans le premier cas, il nous a semblé percevoir une certaine crainte de se compromettre, dans le deuxieme voici ce qu'on nous a répondu : *C'est comme si j"avais eu un gros ac cident et que vous me demandiez de revivre avec vous ma douleur et mes fractures; avec tout l'argent quat m'a fait perdre, je ne veux plus penser au casinos.

Bien entendu, il serait beaucoup trop fastidieus pour le lecteur de lui présenter icil les résultats bruts de tous nas travaux d'observation et d'enquěte. Nous pouvons toutefois en dégager les grandes lignes et il nous parait important à cet égard de souligner la remarquable constance dess observations et propos recueillis tout au long de ces tra. vaux.

Première constatation : les deux clientêles sont incampotibles at la clientèle du casino nuit à la qualité du séjour de la clientèle traditionnelle et a tendance à faire fuir cette dernière.

Deuxième constatation : la clientèle du casino consomme trés peu le produit traditionnel de Charlevoix.

Troisiame constatation : les entreprises touristiques traditionnelles subissent une baisse de leurs revenus, au point de compromettre leur rentabilité.

Quatrieme constatation: on juge que la promotion de Charlevoix mat bea acoup trop l'accent sur la cassino ot nuit à l'image de la règion.

Pour conclure cet exposé méthodolagique, disons que nos travaux dquivalent a une pró-enquéte en vue d'une recherche qui s'impose et qui tarde à venir. Ils suffisent copendant àlegitimer nos interrogations relatives a l'incidence du c asino sur lind ustrie touristique de la région car nous avons respecte la premiere exigence de toute recherche: poser des questions.

Comme nous l'enseigne Madeleine Grawit, dans les deux démarches de la pensée, la déduction et l'induction, ala penste prend un riscue, btablit un compromis entre rigueur et invention... l'impartant $c^{r}$ est de discerner l'essential, d'abstraire, de gènéraliser, de créer des conceptsw'.

1) Grawitz, Madeleine (19815, Mathodes der ceiences soclales. collection : Anćcis Dalloxs (Paris: Dalloc, cinquiènse édition). p. 21 